\title{
Cosmological Data Could have a microscopic, not Macroscopic, Cause
}

\author{
Emmanuil Manousos
}

Astrophysics Laboratory, Department of Physics, National and Kapodistrian

University of Athens, Panepistimiopolis, GR 15783 Zographos, Athens, Greece

\begin{abstract}
A slight continuous increase of the rest masses and the electric charges of material particles can justify quantum phenomena and the cosmological data. This increase can only occur in a strictly defined way: the law of selfvariations. The law of selfvariations condenses into one equation the totality of the cosmological data, as we observe them from the time of Hubble up to the present. The consequences of the selfvariations are recorded persistently and in the most immediate way in the cosmological data. The observation of the redshift of distant astronomical objects by Hubble in the beginning of the last century, leads to only one certain conclusion: one or more physical quantities, which we would expect to be constant in the Universe, in reality vary. The physical theories of the twentieth century justify the redshift macroscopically, via the expansion of the Universe. But the redshift can also be justified differently, with microscopic causes that predict a self-consistent cosmological model in accordance with all of the cosmological data. The problems caused by modern observations to the Standard Cosmological Model, are exactly focused at the points where the two models make different predictions. We propose a revaluation of the cosmological data, based on the law of the selfvariations.
\end{abstract}

Keywords: Redshift, MRB, Supernovae, Early Universe

\section{INTRODUCTION}

The law of selfvariations results from a study we conducted in the microcosm, concerning fields and quantum phenomena. However, when we apply it to the macrocosm it results in an equation with one unknown, which contains as information and justifies, the whole of the cosmological data: the redshift of distant astronomical objects, the cosmic microwave background radiation, the increased luminosity distances of type Ia supernovae, the flatness of the Universe, the fact that the Universe went through a phase of atomic ionization, the anisotropies observed in the microwave background radiation, the existence of dark matter, a very slight variation of the fine-structure constant, the arrow of time in the macrocosm and its nullification in the microcosm.

\section{THE REDSHIFT OF DISTANT ASTRONOMICAL OBJECTS}

In the macrocosm, the law of selfvariations (Manousos, 2013) can be expressed via equation:

$$
\left(\mathrm{m}_{0} \mathrm{c}^{2}+\mathrm{i} \hbar \frac{\dot{\mathrm{m}}_{0}}{\mathrm{~m}_{0}}\right)^{\square}=0
$$

The only unknown being the rest mass $m_{0}$ of material particles. This equation contains as information the redshift of distant astronomical objects in a static Universe. Furthermore, it informs us that gravity can lead the Universe neither to collapse nor to expansion. Therefore, there is no question of solving Equation (1) in an expanding Universe. Another piece of information provided by the equation, is that the total energy content of the Universe is zero. Consequently, the Universe is flat. So, we solve (Manousos, 2013) Equation (1) in a static and flat Universe.

From the solution of Equation (1) we obtain Equation (2):

$$
\mathrm{m}_{0}(\mathrm{r})=\mathrm{m}_{0} \frac{1-\mathrm{A}}{1-A \mathrm{e}^{-\frac{\mathrm{kr}}{\mathrm{c}}}}
$$

where, $\mathrm{m}_{0}(\mathrm{r})$ is the rest mass of a material particle positioned at a distance $r$ from Earth and $m_{0}$ is the 
laboratory value of the rest mass of the material particle on Earth "now". Parameter $\mathrm{k}$ is a positive constant and $\mathrm{c}=3 \times 10^{5} \frac{\mathrm{km}}{\mathrm{s}}$ is the speed of light in vacuum. Parameter A obeys the inequality Equation (3):

$$
\frac{\mathrm{Z}}{1+\mathrm{z}}<\mathrm{A}<1
$$

For every value of the redshift $Z$. Furthermore, it increases at an extremely slow rate with the passage of time t, according to Equation (4):

$$
\frac{\mathrm{dA}}{\mathrm{dt}}=\dot{\mathrm{A}}=\mathrm{kA}
$$

Between the parameters k,A and the Hubble parameter $\mathrm{H}$, the following relation holds Equation (5):

$$
\mathrm{H}=\frac{\mathrm{kA}}{1-\mathrm{A}}
$$

The emission of the electromagnetic spectrum we receive "now" on Earth from an astronomical object at a distance $r$ from Earth, took place before a time interval $\Delta \mathrm{t}=\frac{\mathrm{r}}{\mathrm{c}}$. Therefore, the rest mass $\mathrm{m}_{0}(\mathrm{r})$ of the electron in the astronomical object, at the moment of the emission of the electromagnetic spectrum, was smaller than the corresponding laboratory rest mass $\mathrm{m}_{0}$ of the electron "now" on Earth. As a consequence, the wavelength $\lambda$ of the linear spectrum we receive on Earth is larger than the corresponding laboratory $\lambda_{0}$. By performing the necessary calculations (Manousos, 2013) we obtain the redshift $\mathrm{z}=\frac{\lambda-\lambda_{0}}{\lambda_{0}}$ of the astronomical object from equation:

$$
\mathrm{z}=\frac{1-\mathrm{A} \mathrm{e}^{-\frac{\mathrm{kr}}{\mathrm{c}}}}{1-\mathrm{A}}-1
$$

From Equation (6) we obtain the distance $r$ of the astronomical object as a function of the redshift $\mathrm{z}$ :

$$
\mathrm{r}=\frac{\mathrm{c}}{\mathrm{k}} \ln \left(\frac{\mathrm{A}}{\mathrm{A}-\mathrm{z}(1-\mathrm{A})}\right)=\frac{\mathrm{c}}{\mathrm{H}} \frac{\mathrm{A}}{1-\mathrm{A}} \ln \left(\frac{\mathrm{A}}{\mathrm{A}-\mathrm{z}(1-\mathrm{A})}\right)
$$

According to Equation (3), parameter A takes values close to 1 with $\mathrm{A}<1$. For $\mathrm{A} \rightarrow 1^{-}$, Equation (7) gives (Manousos, 2013) Hubble's law:

$$
\mathrm{r}=\frac{\mathrm{c}}{\mathrm{H}} \mathrm{z}
$$

Practically, for A = 0.999 Equation (7) and (8) are identical.

Figure 1 depicts the graph of $r=r(z)$ for $A=0.900$, $\mathrm{A}=0.950, \mathrm{~A}=0.990, \mathrm{~A}=0.999$, up to $\mathrm{z}=1.5$. As we increase the value of parameter $A$, the curve $r=r(z)$ tends to a straight line, thus approximating Hubble's law.

\section{THE LUMINOSITY DISTANCES R OF DISTANT ASTRONOMICAL OBJECTS WILL ALWAYS BE MEASURED GREATER THAN THEIR REAL DISTANCES $r$}

A consequence of the smaller rest masses of material particles in the past, is the production of smaller amounts of energy during hydrogen fusion and in every mass-toenergy conversion in general. Therefore, the energy powering distant astronomical objects is smaller than expected, resulting in the decrease of their expected intrinsic luminosity. Because of this, the luminosity distances $\mathrm{R}$ of distant astronomical objects will always be measured greater than their real distances r. After the necessary calculations (Manousos, 2013), the following relation is obtained:

$\mathrm{R}=\mathrm{r} \sqrt{1+\mathrm{z}}$

Between the distances Rand r.

Combining Equation 7 and 9 we get the luminosity distance $R$ as a function of the redshift $Z$ :

$R=\frac{c}{H} \frac{A}{1-A} \sqrt{1+z} \ln \left(\frac{A}{A-z(1-A)}\right)$

For every distant astronomical object. Combining Hubble's law, as given by Equation (8), with Equation (9), we get:

$\mathrm{R}=\frac{\mathrm{c}}{\mathrm{H}} \mathrm{z} \sqrt{1+\mathrm{z}}$ 


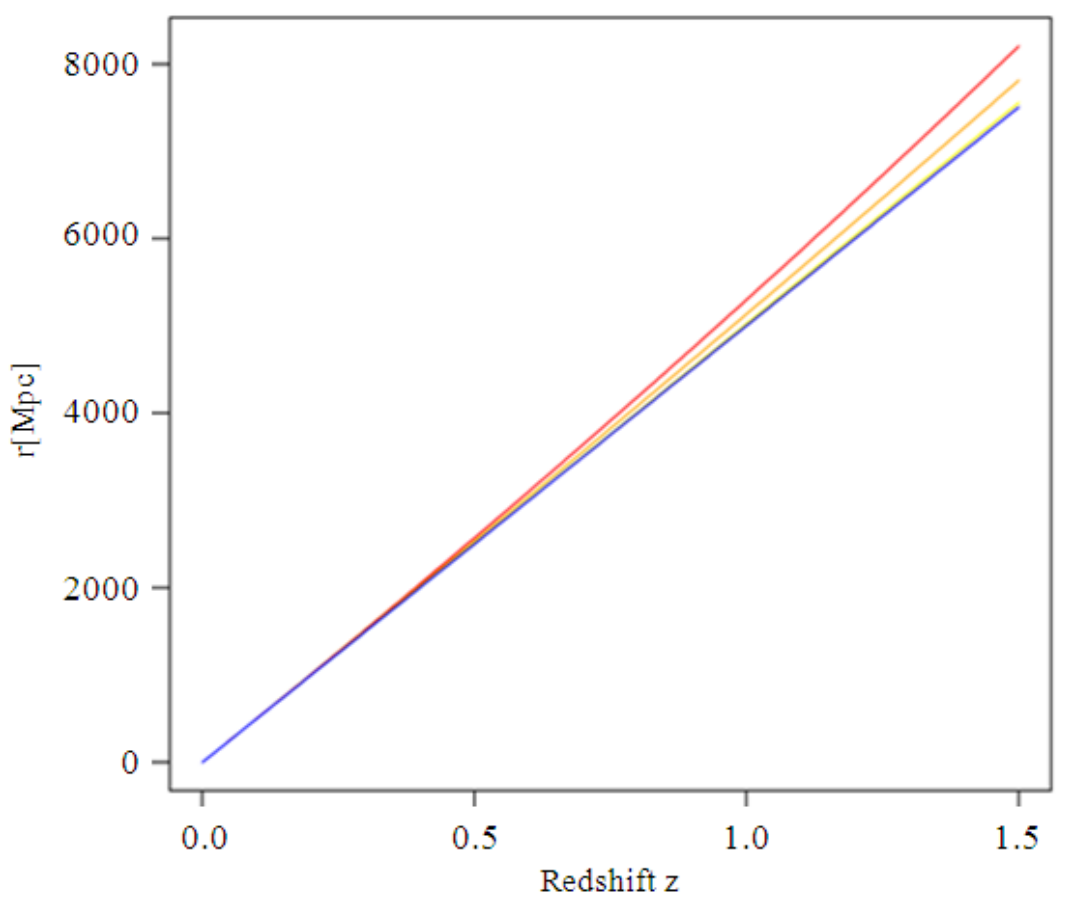

Fig. 1. The graph of $r=r(z)$ for $A=0.900, A=0.950, A=0.990, A=0.999$, up to $z=1.5$. As we increase the value of parameter A, the curve $r=r(z)$ tends to be a straight line, thus approximating Hubble's law

Equation (11) results from Equation (10) for $\mathrm{A} \rightarrow 1^{-}$, exactly as Equation (8) results from Equation (7).

Figure 2 shows the graph of function $\mathrm{R}=(\mathrm{z})$ as given by Equation (10), up to $z=1.5$. For Hubble's parameter we have used the value $\mathrm{H}=60 \frac{\mathrm{km}}{\mathrm{sMpc}}$, close to what we expect (Manousos, 2013) its actual value to be. The most recent measurements, by the Planck satellite, already give a value below $70 \frac{\mathrm{km}}{\mathrm{sMpc}}$.

Figure 3 shows the graph of function $R=R(z)$, as given by Equation (11), up to $Z=1.5$. This graph is recorded in the cosmological data during the last 20 years, through the measurement of the luminosity distances of type Ia supernovae.

Type Ia supernovae are cosmological objects for which we can measure the luminosity distance at great distances. Both the initial measurements (Riess et al., 1998; Perlmutter et al., 1999), as well as subsequent ones (Peacock et al., 2001), confirm the increased luminosity distances of type Ia supernovae, compared to the expected ones. These measurements forced the hypothesis of dark energy onto the standard cosmological model. Several researchers attempt to justify the above measurements in more conventional ways, such as the hypothesis that we live in a region of the Universe which is moving with great velocity with respect to the rest, or other similar ideas. In reality, the increased luminosity distances of type Ia supernovae, record in the cosmological data the decreased, compared to the laboratory ones, rest masses of material particles in distant astronomical objects. Of course, this is also recorded in the redshift of distant astronomical objects. Only, the redshift is justified by the standard cosmological model via the expansion of the Universe, something plausible according to the physical theories of the last century.

In demonstrating the proof (Manousos, 2013) of Equation (10) and (11) we have not considered a change in the volume and therefore, the emission surface of supernovae to very large distances. This is possible due to the multiple consequences of the selfvariations in the evolution of cosmological objects. For large values of redshift $\mathrm{z}$, a deviation of the observations from the predictions of Equation (10) and (11) is likely. Therefore it is important that for $\mathrm{Z}<1.5$ the experimental data strictly confirm the consequences of the diminished rest masses of distant astronomical objects. 
Emmanuil Manousos / American Journal of Space Science 1 (1): 9-21, 2013

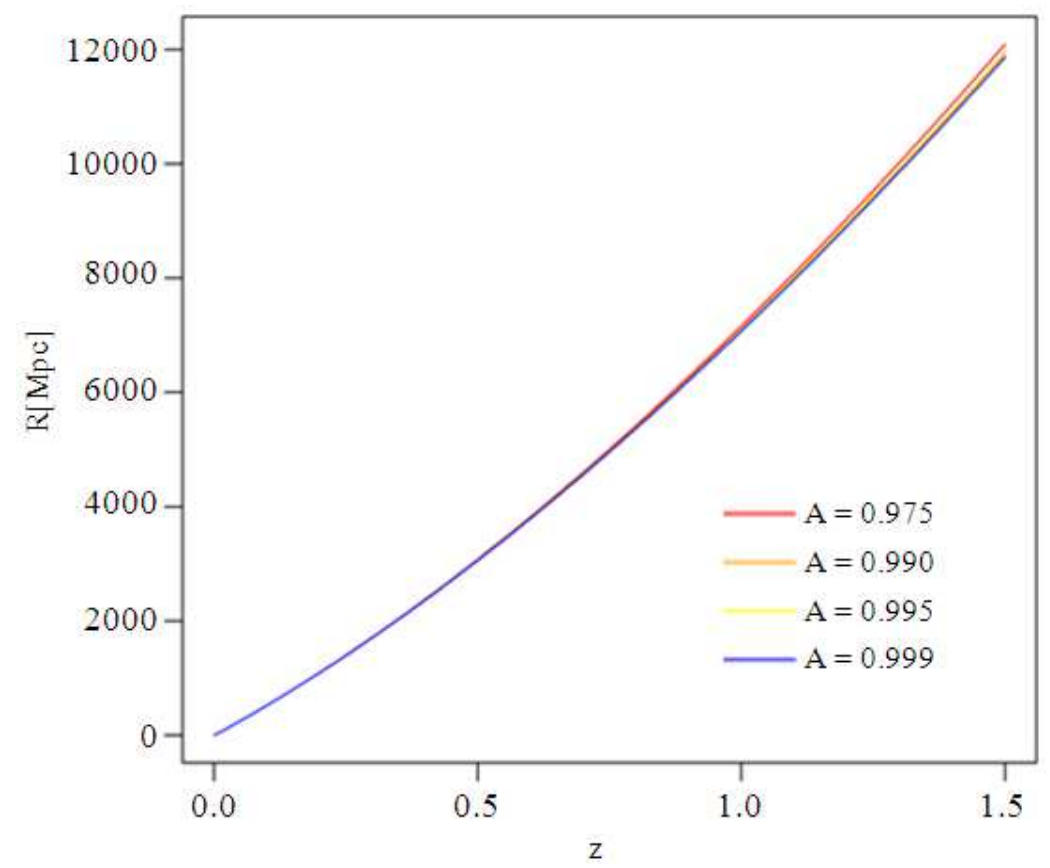

Fig. 2. The graph of the luminosity distance $R=R(z)$ of an astronomical object as a function of the redshift $z$, for $A=0.900, A=$ $0.950 \mathrm{~A}=0.990, \mathrm{~A}=0.999$, up to $\mathrm{z}=1.5$. The measurement of the type Ia supernovae luminosity distances confirms the theoretical prediction of the law of selfvariations.

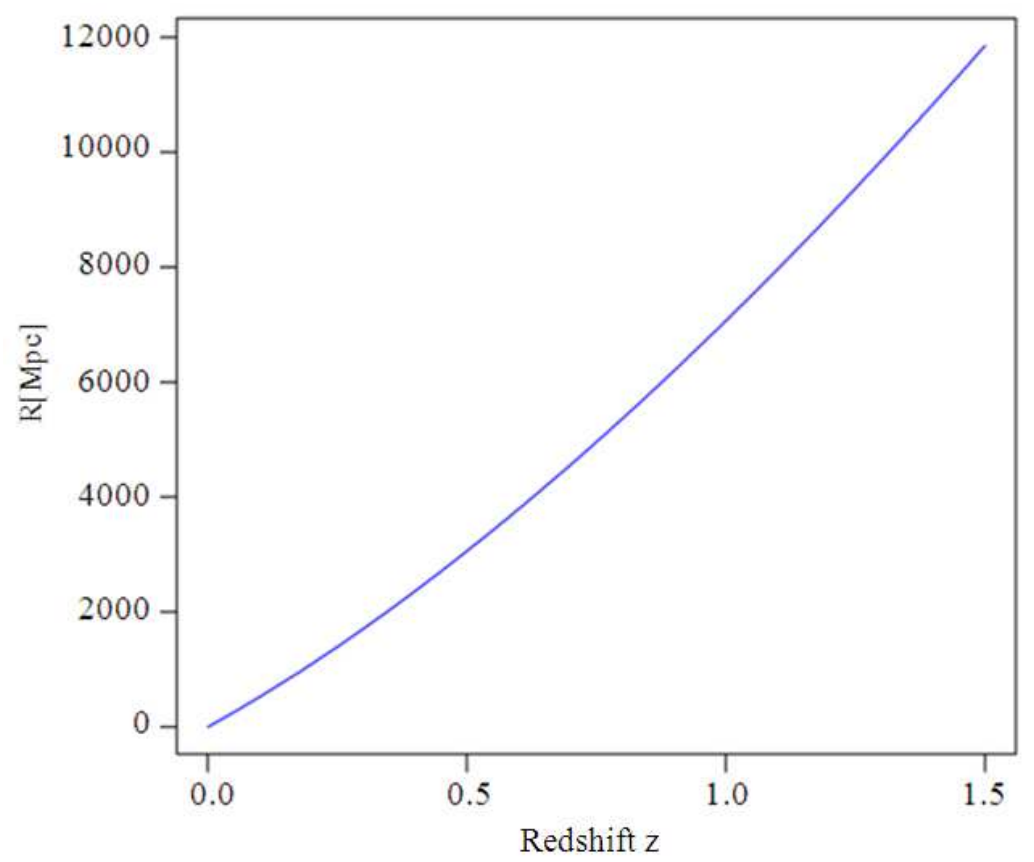

Fig. 3. The theoretically predicted graph of the luminosity distance $R=R(z)$ as a function of the redshift $z$ of astronomical objects. The recording of the above diagram in the cosmological data during the measurement of the luminosity distances of type Ia supernovae, led to the introduction of the concept of dark energy into the framework of the standard cosmological model. 


\section{GRAVITY CANNOT PLAY THE ROLE ATTRIBUTED TO IT BY THE STANDARD COSMOLOGICAL MODEL}

The cosmological model that emerges from the law of selfvariations is self-consistent. The gravitational interaction cannot play the role attributed to it by the standard cosmological model because of the smaller, compared to the laboratory, rest masses of the material particles in distant astronomical objects.

Combining Equation (2) and (6) we obtain:

$$
\frac{\mathrm{m}_{0}(\mathrm{z})}{\mathrm{m}_{0}}=\frac{1}{1+\mathrm{z}}
$$

From this equation for $z=9$ we get $\frac{\mathrm{m}_{0}(\mathrm{z})}{\mathrm{m}_{0}}=0.1$. The strength of the gravitational interaction exerted by an astronomical object with redshift $z=9$ on our galaxy, is only $10 \%$ of the expected one. For even larger values of the redshift $\mathrm{Z}$ the strength of the gravitational interaction practically vanishes. Therefore, gravity cannot lead the Universe either to expansion, or to collapse.

Solving Equation (5) with respect to $\frac{\mathrm{k}}{\mathrm{c}}$, we get:

$\frac{\mathrm{k}}{\mathrm{c}}=\frac{1-\mathrm{A}}{\mathrm{A}} \frac{\mathrm{H}}{\mathrm{c}}$

For $\mathrm{H}=60 \frac{\mathrm{km}}{\mathrm{sMpc}} \mathrm{A}=0.999$ and $\mathrm{c}=3 \times 10^{5} \frac{\mathrm{km}}{\mathrm{s}}$ we get Equation (14):

$$
\frac{\mathrm{k}}{\mathrm{c}}=2 \times 10^{-7} \frac{1}{\mathrm{Mpc}}
$$

Replacing this value of $\frac{\mathrm{k}}{\mathrm{c}}$ into Equation (2) we get:

$$
\frac{\mathrm{m}_{0}(\mathrm{r})}{\mathrm{m}_{0}}=\frac{0.001}{1-0.999 \mathrm{e}^{-2 \times 10^{-7} \mathrm{r}}}
$$

where, the distance $r$ is measured in Mpc.

For values of $r$ of the order of magnitude of $\mathrm{kpc}$, Equation (15) gives $\frac{\mathrm{m}_{0}(\mathrm{r})}{\mathrm{m}_{0}}=1$. Therefore, the strength of the gravitational interaction is not affected at the scale of galactic distances. For example, taking $\mathrm{r}=100 \mathrm{kpc}$, Equation (15) gives $\frac{\mathrm{m}_{0}(\mathrm{r})}{\mathrm{m}_{0}}=0.99999$. The selfvariations affect the strength of the gravitational interaction for distances of the order of Mpc. For $r=100 \mathrm{Mpc}$ we have $\frac{\mathrm{m}_{0}(\mathrm{r})}{\mathrm{m}_{0}}=0.98$, whereas for $\mathrm{r}=2 \times 10^{3} \mathrm{Mpc}$ we get $\frac{\mathrm{m}_{0}(\mathrm{r})}{\mathrm{m}_{0}}=0.7145$. The strength of the gravitational interaction exerted by an astronomical object at a distance of $r=2000 \mathrm{Mpc}$ on our galaxy is only $71,45 \%$ of the expected.

We therefore conclude that, due to the selfvariations, gravity weakens at cosmological distances. Gravity dominates on a local level, at scales of a few hundreds or thousands of $\mathrm{kpc}$.

We note here that for different values of the parameter A, from those permitted by inequality (3), other numerical values result in Equation (15). This is because of Equation (13). However, the ratio $\frac{\mathrm{m}_{0}(\mathrm{r})}{\mathrm{m}_{0}}$ does not depend on the value we use for parameter A. This is expected, since according to Equation (12) it is:

$$
\frac{\mathrm{m}_{0}(\mathrm{r})}{\mathrm{m}_{0}}=\frac{\mathrm{m}_{0}(\mathrm{z})}{\mathrm{m}_{0}}=\frac{1}{1+\mathrm{z}}
$$

The ratio $\frac{\mathrm{m}_{0}(\mathrm{r})}{\mathrm{m}_{0}}$ is uniquely determined by the value of the redshift $\mathrm{Z}$.

\section{THE VERY EARLY UNIVERSE}

All the equations of the cosmological model predicted by the law of the selfvariations are compatible with the condition $r \rightarrow \infty$. These equations are expressed in such a way that this condition provides information about the very early Universe; about the initial state of the Universe.

For $\mathrm{r} \rightarrow \infty$. Equation (2) gives Equation (16):

$\mathrm{m}_{0}(\mathrm{r} \rightarrow \infty) \rightarrow \mathrm{m}_{0}(1-\mathrm{A})$

The inequality (3), $\frac{\mathrm{Z}}{1+\mathrm{z}}<\mathrm{A}<1$, holds for every $\mathrm{z}$, therefore $\mathrm{A} \rightarrow 1^{-}$. Thus, from relation (16) we infer that the initial form of the Universe, only slightly differs from the vacuum. 
The increase of the rest masses of the material particles with the passage of time destroys the initial uniformity and immobility, brings about the first infinitesimal motions of the particles and shifts the system ever so slightly above the temperature of $0 \mathrm{~K}$ (temperature expresses the kinetic state of the system's particles). Although the initial state of the Universe only slightly differs from the vacuum, the system is dynamic and temporally variable due to the selfvariations. The evolution of the selfvariations with the passage of time led the Universe to the form we observe it today. This prediction is confirmed by the calculations that follow in the next paragraphs.

\section{THE ORIGIN OF THE COSMIC MICROWAVE BACKGROUND RADIATION}

The law of selfvariations predicts (Manousos, 2013) that, in distant astronomical objects, the Thomson scattering coefficient $\sigma_{\mathrm{r}}(\mathrm{r})$ and the Klein-Nishina scattering coefficient $\sigma(\mathrm{r})$ are larger than their laboratory values $\sigma_{\mathrm{r}}$ and $\sigma$, respectively. The following Equation (17 and 18) are predicted:

$$
\begin{aligned}
& \frac{\sigma_{\mathrm{T}}(\mathrm{r})}{\sigma_{\mathrm{T}}}=\frac{\sigma(\mathrm{r})}{\sigma}=\left(\frac{1-\mathrm{Ae}^{-\frac{\mathrm{kr}}{\mathrm{c}}}}{1-\mathrm{A}}\right)^{2} \\
& \frac{\sigma_{\mathrm{T}}(\mathrm{z})}{\sigma_{\mathrm{T}}}=\frac{\sigma(\mathrm{z})}{\sigma}=(1+\mathrm{z})^{2}
\end{aligned}
$$

They quantify the dependence of the scattering coefficients on the distance $r$ and the redshift $\mathrm{z}$ of an astronomical object.

For very large distances $r(r \rightarrow \infty)$, i.e., in the very early Universe, Equation (17) gives:

$$
\frac{\sigma_{\mathrm{T}}(\mathrm{r} \rightarrow \infty)}{\sigma_{\mathrm{T}}}=\frac{\sigma(\mathrm{r} \rightarrow \infty)}{\sigma}=\frac{1}{(1-\mathrm{A})^{2}}
$$

From inequality (3) it follows that $\mathrm{A} \rightarrow 1^{-}$. Therefore, from Equation (19) it follows that in the very early Universe the Thomson and Klein-Nishina scattering coefficients have enormous values. Theoretically they become infinite, rendering the very early Universe opaque. The cosmic microwave background radiation originates from this phase of the evolution of the Universe. We remind that, as already stated in the previous paragraph, the temperature of the Universe at this phase is close to $0 \mathrm{~K}$. Furthermore, the cosmic microwave background radiation, as a consequence of the enormous values of the scattering coefficients in the very early Universe, originates from the totality of the space occupied by the Universe.

\section{THE UNIVERSE IS FLAT}

From relation (16):

$$
\mathrm{m}_{0}(\mathrm{r} \rightarrow \infty) \rightarrow \mathrm{m}_{0}(1-\mathrm{A})
$$

And inequality (3):

$$
\frac{\mathrm{z}}{1+\mathrm{z}}<\mathrm{A}<1
$$

Which holds for every value of the redshift $z$, we conclude that the total energy-content of the very early Universe tends to zero:

$$
\mathrm{m}_{0} \mathrm{c}^{2}(\mathrm{r} \rightarrow \infty) \rightarrow \mathrm{m}_{0} \mathrm{c}^{2}(1-\mathrm{A}) \rightarrow 0
$$

The law of the selfvariations is in accordance with the principle of the conservation of energy (Manousos, 2013). Therefore, in all phases of the evolution of the Universe, including today, its total energy-content is zero. Consequently, the Universe is flat.

The COBE, WMAP and Planck satellite missions have successively confirmed the flatness of the Universe.

\section{THE LARGE-SCALE STRUCTURES OF THE UNIVERSE}

The law of selfvariations at the limit, in the very far past, predicts the vacuum as the beginning of the Universe. All points within the Universe are initially equivalent and the Universe originates "from everywhere", like the cosmic microwave background radiation (paragraph 6).

The mathematical equations that describe the law of selfvariations predict (Manousos, 2013) an intermediate state between matter and the photon. This intermediate state justifies the quantum phenomena and, in the exact same way, predicts the destruction of the absolute homogeneity of the vacuum in the very early Universe. This destruction of the absolute homogeneity of the very early Universe can take place in a small region of space, or at enormous distances in the order of $10^{9}$ ly or even larger. This explains the monstrous webs of matter within vast expanses of empty space we observe (Gott et al., 2005) with modern observational instruments. 
The increase of the rest masses with the passage of time, due to the selfvariations, strengthens the gravitational interaction and accumulates matter in various directions. The consequences of the accumulation of matter depend on the initial volume occupied by and on the amount of matter contained within, the region where this accumulation takes place. In every case, the total initial energy of the accumulating matter is zero, according to relation (16).

Within the dimensions of a galaxy, the accumulation of matter increases the temperature through the conversion of gravitational potential energy to heat. A percentage of the particles of matter accumulates in an initial core of ever increasing temperature. Nevertheless, a sufficient percentage of particles remains distributed within the initial space, around the hot core, because of the extremely slow rates of evolution of the selfvariations and strengthening of gravity.

Further accumulation within the first core will lead to the creation of a second core, until the temperature reaches values that permit nuclear fusion. The initialization of nuclear fusion stops the further accumulation of matter.

We separated the process of accumulation into two phases and mentioned two cores, for the following reason: The amount of matter left outside the first core in the initial phase of accumulation is at a low temperature, slightly above $0 \mathrm{~K}$. However, the amount of matter which stays outside the second, real core, is already at high temperature. If we take into account the very high value of the Reynolds coefficient, turbulence will emerge within this region. Therefore, stars will form in this region. A further conclusion that emerges from the process of the accumulation is that the density of matter in the central core will be greater than in the rest of the galaxy.

In reality, the process of the accumulation is not separated into phases, but evolves continuously from its start, until the formation of the real astronomical object we observe today. This object can be a galaxy or a cluster of galaxies, depending on the amount of the accumulating matter.

The law of selfvariations provides a sufficient amount of information about the initial state of the region where matter accumulates and also about the rate of strengthening of gravity. Therefore the process is amenable to computer simulation. This is the most appropriate way in which to draw safe conclusions about the distribution of matter in the large-scale structures of the Universe predicted by the law of selfvariations.

The time interval of the order of $80 \times 10^{9} \mathrm{yr}$, needed for the formation of the Sloan Great Wall (Gott et al., 2005) is much larger than the age of $13,8 \times 10^{9}$ yr predicted for the Universe by the standard cosmological model.
Furthermore, modern observational instruments have found the existence of galaxies very close to the point at which the Big Bang is placed by the standard cosmological model. We propose a detailed study of whether the existence of galaxies so close to the Big Bang is in agreement with the standard cosmological model. We predict that improved observational instruments will detect galaxies at even greater distances, with even greater redshifts, from the ones we observe today.

\section{THE SELFVARIATION OF THE ELECTRIC CHARGE PREDICTS A SLIGHT VARIATION OF THE FINE- STRUCTURE CONSTANT}

Equation (1) predicts the totality of the cosmological data, as we observe them, except for a very slight variation for the fine-structure constant predicted by the law of selfvariations. This variation is predicted by the variation of the electric charge.

The electric charge $\mathrm{q}(\mathrm{r})$ of the electron in an astronomical object at distance $r$ from Earth, is different from the electric charge $q$ of the electron in the laboratory (Manousos, 2013), according to Equation (20):

$$
q(r)=q \frac{1-B}{1-B e^{-k 1 \frac{r}{c}}}
$$

Regarding parameter B we know that Equation (21):

and that it varies slightly with the passage of time $t$, according to Equation (22):

$$
\frac{\mathrm{dB}}{\mathrm{dt}}=\dot{\mathrm{B}}=\mathrm{k}_{1} \mathrm{~B}
$$

The fine-structure constant a Equation (23):

$$
\alpha=\frac{\mathrm{q}^{2}}{4 \pi \varepsilon_{\mathrm{o}} \mathrm{ch}}
$$

Depends on the electric charge $\mathrm{q}$ and is, therefore, affected by the selfvariations. If we denote by a(r) the value the fine-structure constant at an astronomical object at a distance $r$ from Earth, we get:

$$
\frac{\alpha(r)}{\alpha}=\left(\frac{q(r)}{q}\right)^{2}
$$


and with Equation (20) we see that:

$$
\frac{\alpha(r)}{\alpha}=\left(\frac{q(r)}{q}\right)^{2}=\left(\frac{1-B}{1-B e^{-k_{1} \frac{r}{c}}}\right)^{2}
$$

The fact that electric charge exists in pairs of opposite quantities, allows for the evolution of the selfvariations of the electric charge in two directions: either towards increase or towards decrease of the absolute value $|\mathrm{q}|$ of the electric charge (Manousos, 2013), something which is not the case for the rest mass. The two different directions of evolution of the selfvariation of the electric charge can be expressed by the sign of constant $\mathrm{k}_{1}$. For $\mathrm{k}_{1}>0$ the electric charge increases in absolute value and $\mathrm{k}_{1}<0$ for it decreases in absolute value.

From Equation (24) we get that for $\mathrm{k}_{1}>0$ it is Equation (25):

$$
\frac{\alpha(\mathrm{r})}{\alpha}<1
$$

whereas for $\mathrm{k}_{1}<0$ it is Equation (26):

$$
\frac{\alpha(\mathrm{r})}{\alpha}>1
$$

The recording, in the cosmological data, of a very slight variation of the fine-structure constant (Webb et al., 2011) confirms the selfvariation of the electric charge. This recording is of the same importance as the recording of the increased luminosity distances of type Ia supernova (Riess et al., 1998; Perlmutter et al., 1999), which confirms the selfvariation of the rest masses.

From the observational data (Webb et al., 2011; 1999; Tzanavaris et al., 2005) we know that the selfvariation of the electric charge evolves as a much slower rate than the selfvariation of the rest mass. We predict that improvements in the observational instruments will definitively confirm the variation of the fine-structure constant as a consequence of the variation of the electric charge of the electron.

\section{THE DECREASE OF ATOMIC IONIZATION ENERGIES AT DISTANT ASTRONOMICAL OBJECTS}

The ionization and excitation energy of atoms is proportional to the factor $m_{o} q^{4}$, where $m_{o}$ is the rest mass and $\mathrm{q}$ is the electric charge of the electron. Thus, we have Equation (27):

$$
\frac{X_{n}(r)}{X_{n}}=\frac{m_{o}(r)}{m_{o}}\left(\frac{q(r)}{q}\right)^{4}
$$

and due to the extremely slow rate of change of the electric charge we get:

$$
\frac{X_{n}(r)}{X_{n}}=\frac{m_{o}(r)}{m_{o}}
$$

where, $X_{n}$ is the excitation or ionization energy in the laboratory and $X_{n}(r)$ is the corresponding energy in an astronomical object at a distance $r$ from Earth.

Combining Equation (28) and (2) we get:

$\frac{X_{n}(r)}{X_{n}}=\frac{1-A}{1-A e^{-k \frac{r}{c}}}$

while combining Equation (28) and (12) we get:

$\frac{X_{n}(r)}{X_{n}}=\frac{1}{1+z}$

According to Equation (29) and (30) the redshift $\mathrm{z}$ affects the degree of ionization at distant astronomical objects. Boltzmann's formula:

$\frac{\mathrm{N}_{\mathrm{n}}}{\mathrm{N}_{1}}=\frac{\mathrm{g}_{\mathrm{n}}}{\mathrm{g}_{1}} \mathrm{e}^{-\frac{\mathrm{X}_{\mathrm{n}}}{\mathrm{KT}}}$

Gives the multitude of excited atoms $\mathrm{N}_{\mathrm{n}}$, at energy level $\mathrm{n}$, on a stellar surface at thermodynamic equilibrium. With $\mathrm{X}_{\mathrm{n}}$ we denote the excitation energy from level 1 to level $\mathrm{n}, \mathrm{T}$ is the temperature of the stellar surface in Kelvin, $\mathrm{K}=1,38 \times 10^{-23} \frac{\mathrm{J}}{\mathrm{K}}$ is Boltzmann's constant and $g_{n}$ is the degree of multiplicity of energy level $\mathrm{n}$ (that is, the number of energy levels in which it splits in a magnetic field).

Combining Equation (30) and (31) we obtain:

$\frac{N_{n}}{N_{1}}=\frac{g_{n}}{g_{1}} e^{-\frac{X_{n}}{K T(1+z)}}$

For the distant astronomical object. In the case of hydrogen, $\mathrm{n}=2, \mathrm{X}_{2}=10.15 \mathrm{eV}=16.24 \times 10^{-19} \mathrm{~J}, \mathrm{~g}_{1}=2$, 
$\mathrm{gg}_{2}=8$ and for a temperature of the solar surface $\mathrm{T}=$ $6000 \mathrm{~K}$, Equation (31) gives that only one atom in $10^{8}$ is in the $\mathrm{n}=2$ state. For the same temperature, Equation (32) gives that for $\mathrm{z}=1$ it is $\frac{\mathrm{N}_{2}}{\mathrm{~N}_{1}}=2.2 \times 10^{-4}$, for $\mathrm{z}=2$ it is $\frac{\mathrm{N}_{2}}{\mathrm{~N}_{1}}=5.8 \times 10^{-3}$ and that for $\mathrm{z}=5$ it is $\frac{\mathrm{N}_{2}}{\mathrm{~N}_{1}}=0.15$.

According to Equation (18) the opacity of stellar surfaces increases at distant astronomical objects, while, according to Equation (32), the degree of atomic ionization also increases. These two factors have opposite consequences for the electromagnetic radiation of distant astronomical objects. We propose an analytical study regarding the consequences of this antithesis.

From Equation (29) and for large distances $(r \rightarrow \infty)$ we get Equation (33):

$$
\frac{X_{n}(r \rightarrow \infty)}{X_{n}} \rightarrow 1-A
$$

Taking into account inequality (3), we conclude from relation (33) that in the very early Universe the ionization energies tend to zero. Therefore, the Universe went through a phase of ionization (Spergel et al., 2007) during its evolution.

According to Equation (29) and (32), over very long distances beyond the limits of the observable universe, matter is ionized. Thus we expect the existence of an electromagnetic noise from this area of the universe. The Equation (2) and (20) enable us to predict the properties of this electromagnetic noise. We recommend the construction of a suitable experiment to record this noise in the cosmological data. The detailed study for this phase in the evolution of the Universe will be presented in a different article.

\section{THE DECREASE OF COHESIVE FORCES OF PARTICLES IN DISTANT ASTRONOMICAL OBJECTS}

A measure of the cohesion of composite particles, of the strength of the nuclear interaction, is the amount of rest mass $\Delta \mathrm{m}_{\mathrm{o}}$ of the elementary particles comprising the composite particle, that is converted into binding energy $\Delta \mathrm{m}_{\mathrm{o}} \mathrm{c}^{2}$. According to Equation (2), the binding energy of the particles $\Delta \mathrm{m}_{\mathrm{o}}(\mathrm{r}) \mathrm{c}^{2}$ is smaller at distant astronomical objects, than the corresponding laboratory energy:

$$
\frac{\Delta \mathrm{m}_{\mathrm{o}}(\mathrm{r}) \mathrm{c}^{2}}{\Delta \mathrm{m}_{\mathrm{o}} \mathrm{c}^{2}}=\frac{1-\mathrm{A}}{1-A \mathrm{e}^{-\mathrm{k} \frac{\mathrm{r}}{\mathrm{c}}}}
$$

The same equation can be written in the form:

$$
\frac{\Delta \mathrm{m}_{\mathrm{o}}(\mathrm{r}) \mathrm{c}^{2}}{\Delta \mathrm{m}_{\mathrm{o}} \mathrm{c}^{2}}=\frac{1}{1+\mathrm{z}}
$$

Taking into account Equation (12).

For large distances $(r \rightarrow \infty)$ Equation (34) gives Equation (36):

$$
\frac{\Delta \mathrm{m}_{\mathrm{o}}(\mathrm{r} \rightarrow \infty) \mathrm{c}^{2}}{\Delta \mathrm{m}_{\mathrm{o}} \mathrm{c}^{2}} \rightarrow 1-\mathrm{A}
$$

According to Equation (34), the binding energies of particles decrease as we move back in time. Taking into consideration inequality (3) we conclude from relation (36) that in the very early Universe the binding energies of particles, for the formation of composite particles, tend to zero. In the very early Universe, at temperatures close to $0 \mathrm{~K}$, composite particles decompose into the individual particles that constitute them.

Enormous quantities of energy are required today in order to break up the particles in the laboratory. But this is a consequence of the evolution of the selfvariations, which strengthened the binding energies of the particles. According to Equation (34) and relation (36), nucleosynthesis can be achieved at very low temperatures, close to $0 \mathrm{~K}$, in the very early Universe. The high energies we measure in the laboratory today are not required during the initial phase of synthesis of composite particles in the very early Universe (Alpher et al., 1948).

The cosmological model of the selfvariations favors the synthesis of composite particles from much smaller particles, quite likely even smaller than the ones we consider elementary today. The strengthening of the cohesive forces of particles with the passage of time, due to the selfvariations, could have rendered composite particles practically inseparable, leaving us no choice but to recognize them as elementary particles in the laboratory. Recognizing this, we can easily justify the existence of dark matter. As we mentioned in paragraph 8 , a percentage of particles remains outside the area of matter accumulation during the formation of the largescale structures in the Universe. These particles remain close to $0 \mathrm{~K}$, whereas the particles that accumulate in the large-scale structures of matter find themselves at progressively higher temperatures. The different conditions could lead to the synthesis of different particles (Walker et al., 2009). Indeed, it is very likely that a basic dark matter particle analogous to the hydrogen of normal matter has formed, together with other derived particles, as is the case with visible matter. 
Regarding the evolution of the Universe, there are points we completely understand, as well as points that demand further investigation on the basis of the theoretical background provided by the law of selfvariations. The masses of supernovae Ia are smaller than expected, resulting in the increase of the luminosity distances we measure for the specific cosmological objects. Another point we can predict is the order in which nuclear reactions take place in the very early Universe. In the laboratory today we know how nuclear reactions are arranged based on the amount of energy required for them to take place. According to Equation (34) and relation (36) this arrangement is the same (Alpher et al., 1948) in the very early Universe predicted by the law of selfvariations. A point which we do not know and which plays an important role in the evolution of the Universe, is the moment at which the electric charge appears in the "luminous" matter. This is a central point of investigation for the understanding of the evolution of the Universe.

In the selfvariation model, the nucleosynthesis of chemical elements occurs in two clearly distinct phases. The first has to do with the very early universe, while the second takes place much later, after the formation of stars, at high temperatures. According to Equation (34) and (35), in the past the cohesion energies of particles were much smaller than the laboratory ones. This can explain the formation of heavier elements in the past inside the sun and stars in general and during the initial formation of large structures of the universe (paragraph 8 ), at temperatures where, until now, we did not consider heavy element nucleosynthesis possible.

\section{THE AGE AND SIZE OF THE UNIVERSE}

All of the equations of the cosmological model resulting from the law of the selfvariations are compatible with the condition $\mathrm{r} \rightarrow \infty$. Consequently, the equations themselves do not pose some limit for the size and age of the Universe. Therefore, we have to examine anew the content of the terms "size" and "age" of the Universe.

Since our equations are compatible with the condition $\mathrm{r} \rightarrow \infty$, what we observe today is only a small part of the Universe. The point at which the standard cosmological model places limits on the Universe, is in reality the point at which matter begins to take its current recognizable form. The question according to the standard cosmological model "what was there before the Big Bang", transforms into the question "what was the form of matter in the very far past, at enormous distances, beyond the limits of the Universe we observe today". We can calculate the value of any parameter for every value of the distance $r$, even for $r \rightarrow \infty$. The answer given by these calculations to the previous question, is that the Universe comes from the vacuum. In reality, an absolutely comprehensible relation emerges between matter and the vacuum.

In paragraph 9 we saw that the selfvariation of the electric charge can proceed in two directions (Manousos, 2013). This capability of the electric charge creates an anisotropy which could be recorded in the cosmological data through the variation of the fine-structure constant (Webb et al., 2011). We propose the investigation of a possible correlation between the temperature anisotropy of the Universe recorded by the Planck satellite and the possibility of the evolution of the selfvariation of the electric charge in two directions. However, the main factor that increases the probability of recording anisotropies in the cosmological data (Clowes et al., 2013) stems from the fact that we only observe a small part of the Universe. We predict that the improvement of observational instruments will record additional anisotropies in the cosmological data due to this factor.

The compatibility of the equations of the model of selfvariations with the condition $r \rightarrow \infty$, means that we can go very far into the past. This is in agreement with the estimation that a large amount of time is required in order for the Universe to reach the form in which we observe it today, starting from a state that only slightly differed from the vacuum.

The amount time which the standard cosmological model regards as the age of the Universe is, in reality, the time interval during which matter has the form we observe today. Cases such as that of the star HD140283, have to be studied in detail. The time (Bond et al., 2013) $(14.5 \pm 0.8) \times 10^{9} \mathrm{yr}$ is the time interval from the creation of the star onwards. The law of selfvariations predicts an even greater time interval until the creation of the star.

With the flatness of the Universe confirmed observationally, the time interval $\mathrm{T}(\mathrm{z})$ required for the electromagnetic radiation from a distant astronomical object to reach Earth, is Equation (37):

$\mathrm{T}(\mathrm{z})=\frac{\mathrm{R}}{\mathrm{c}}>\frac{\mathrm{r}(\mathrm{z})}{\mathrm{c}}$

where, $\mathrm{R}$ the luminosity distance of the astronomical object.

It holds that: 


$$
\mathrm{r}(\mathrm{z})=\frac{\mathrm{cz}}{\mathrm{H}}
$$

according to Hubble's law.

Combining relation (37) and Equation (38) we obtain:

$$
\mathrm{T}(\mathrm{z})>\frac{\mathrm{z}}{\mathrm{H}}
$$

From Equation (39) it follows that Equation (40):

$$
\mathrm{T}(\mathrm{z})>\frac{1}{\mathrm{H}}
$$

For $z>1$. Therefore, the time interval $T(z)$ is greater than the age of the Universe provided by the standard cosmological model. This is the reason why the initial measurements (Riess et al., 1998; Perlmutter et al., 1999) were taken in the direction of proving that the luminosity distances of supernovae are smaller than the ones predicted by Hubble's law. Such outcome would have supported the standard cosmological model. Instead, the luminosity distances were measured much greater than the ones predicted by Hubble's law.

\section{THE ARROW OF TIME IN THE MACROCOSM AND ITS NULLIFICATION IN THE MICROCOSM}

The law of selfvariations contains the arrow of time in the macrocosm. The Universe comes from the vacuum and evolves in a specific direction determined by the law of selfvariations. This evolution is recorded in the cosmological data, since we observe the Universe as it was in the past. The selfvariations constantly "move" the Universe away from the state of the vacuum, but the Universe remains consistent with its origin, having a total energetic content of zero. This consequence finally answers the question posed by Leibniz 300 years ago, "why is there something, instead of nothing".

Applying the law of selfvariations in the microcosm, we find that the arrow of time does not exist. While we are using the same law, the information we obtain is completely different: it informs us about the way in which the rest mass of a material particle is distributed in spacetime (Manousos, 2013). It emerges that Schrödinger's equation and related equations, play a central role in this distribution. The arrow of time that so prominently appears in the macrocosm, does not exist in the microcosm.

\section{THE FUTURE EVOLUTION OF THE UNIVERSE}

The rate of increase of the Hubble parameter and also of the redshift of astronomical objects, is a measure for evaluating the future evolution of the Universe.

The parameter $\mathrm{H}$ of Hubble is predicted (Manousos, 2013) to increase at a rate of:

$$
\frac{\mathrm{dH}}{\mathrm{dt}}=\dot{\mathrm{H}}=\frac{\mathrm{H}^{2}}{\mathrm{~A}}
$$

$$
\text { For } \mathrm{A}=1 \text { and } \mathrm{H}=60 \frac{\mathrm{km}}{\mathrm{sMpc}}=2 \times 10^{-18} \mathrm{~s} \text {, Equation }
$$
gives:

$$
\dot{\mathrm{H}}=4 \times 10^{-36} \mathrm{~s}^{-2}
$$

The redshift $\mathrm{Z}$ of the distant astronomical objects is predicted (Manousos, 2013) to increase at a rate of:

$$
\frac{\mathrm{dz}}{\mathrm{dt}}=\dot{\mathrm{z}}=\frac{\mathrm{H}}{\mathrm{A}} \mathrm{z}
$$

$$
\text { For } \mathrm{A}=1 \text { and } \mathrm{H}=60 \frac{\mathrm{km}}{\mathrm{sMpc}}=2 \times 10^{-18} \mathrm{~s} \text {, Equation (42) }
$$
gives:

$$
\dot{\mathrm{z}}=\mathrm{z} \cdot 6,3 \times 10^{-11} \mathrm{yr}^{-1}
$$

The increase of the Hubble parameter $H$, as well as the increase of the redshift $\mathrm{z}$ with the passage of time, are caused by the increase of parameter A given by Equation (4).

\section{FOR THE TEMPERATURE DIFFERENCE BETWEEN THE NORTHERN AND SOUTHERN HEMISPHERES OF THE UNIVERSE}

The cosmological model of the selfvariations provides two fundamental reasons for the anisotropies recorded in the cosmological data. The first is that we observe only a small part of the universe. Actual isotropy of the universe must be looked for in much larger scales. The second reason is provided from the possibility of the selfvariations of electric charges to evolve into two directions (Manousos, 2013). This fact has already been recorded in the data for the fine structure constant (paragraph 9).

Combing Equation (27), (2) and (20) we get: 


$$
\frac{X_{n}(r)}{X_{n}}=\frac{1-A}{1-A e^{-\frac{k r}{c}}}\left(\frac{1-B}{1-B e^{-\frac{k_{1} r}{c}}}\right)^{4}
$$

For the ionization energy $X_{n}(r)$ of the distant astronomical objects. The very slow evolution of the selfvariations of the electric charge allowed us to approach Equation (43) with Equation (28). Nevertheless the selfvariations of electric charge affect the ionization energy of distant astronomical objects, according with Equation (43). Therefore they affect also the degree of ionization of atoms, in accordance with Equation (31). A greater degree of ionization implies larger numbers of particles and therefore lower temperature as we know from fundamental physics.

From the measurements on the fine structure constant (Webb et al., 2011) we know that during the past its value was smaller from that in the laboratory in the Northern hemisphere of the universe and larger in the Southern. In accordance with Equation (23) this means that in the distant past the electric charge of the electron in the Northern hemisphere was smaller than that in the Southern. Therefore according to Equation (27), the ionization energies of atoms in the Northern hemisphere were smaller than those in the Southern, therefore in accordance with Equation (31) the number of particles in the Northern hemisphere is larger and therefore the temperature of the Northern hemisphere is predicted smaller than that of the Southern. Based on Equation (43) we can carry out a detailed study which also concludes to the same results. This study will be presented in a forthcoming paper.

We propose that the fine structure constant be measured in the direction of the cold spot of the Southern hemisphere of the Universe that was recorded by the Planck satellite. We predict that its value will be measured slightly smaller than in the laboratory.

\section{DISCUSSION}

The standard cosmological model prevailed against other competing models, mainly because it explains three fundamental cosmological data: The redshift of distant astronomical objects, the microwave background radiation and the nucleosynthesis of the atoms of matter. Recent data however, especially from the last fifteen years, do not agree with the predictions of the standard cosmological model (SCM). The anisotropies recorded recently by the Planck satellite, the temperature difference between the North and South hemisphere of the universe, the by now documented change in the fine structure constant, the extraordinary time span estimated that it takes to accumulate the material of the monstrous Sloan wall, the immense distance to which the structure of the 73 quasars observed at the beginning of the year extends, the excessive brightness distances measured for supernovae, are completely contrary to the predictions of the standard cosmological model. Moreover, the SCM has problems with the origin of dark matter and the observed lack of antimatter in the universe.

The cosmological model of selfvariations is consistent with all cosmological data. It predicts the redshift, the microwave background radiation and nucleosynthesis, although the latter requires additional investigation for the completeness of its predictions. This is natural for a new model that is essentially completely different from all the previous ones. It is characteristic that the change of the fine structure constant, the dark matter and the absence of antimatter in the observed universe, are predicted to have a common cause, the selfvariation of the electric charge. This topic will be presented in detail in a separate article. We refer to these to show the sweeping amount of data and information provided by the law of the selfvariations. We may well say that the law of the selfvariations gives the cause of all cosmological data, observed since the era of Edwin Hubble until today. The model of selfvariations clearly excels against the SCM and other models that fundamentally explain the redshift with the expansion of the universe.

\section{CONCLUSION}

We propose a revaluation of the cosmological data, based on the law of the selfvariations. Both in observational level, such as the measurement of the fine structure constant towards the direction of the cold spot identified by the Planck satellite in the southern hemisphere, as well as in theory. This revaluation obviously requires the contribution from many researchers in various fields related to cosmology.

\section{REFERENCES}

Alpher, R.A., H. Bethe and G. Gamow, 1948. The origin of chemical elements. Phys. Rev., 73: 803-804. DOI: 10.1103/PhysRev.73.803 
Bond, H.E., E.P. Nelan, D.A. VandenBerg, G.H. Schaefer and D. Harmer, 2013. HD 140283: A Star in the solar neighborhood that formed shortly after the big bang. Astrophys. J. Lett., 765: L12-29. DOI: 10.1088/2041-8205/765/1/L12

Clowes, R.G., K.A. Harris, S. Raghunathan, L.E. Campusano and I.K. Sochting et al., 2013. A structure in the early universe at $\mathrm{z} \sim 1.3$ that exceeds the homogeneity scale of the R-W concordance cosmology. Monthly Notices Royal Astronomical Soc., 429: 2910-2916.

Gott, J.R., M. Juric, D. Schlegel, F. Hoyle and M. Vogeley et al., 2005. A map of the universe. Astrophys. J., 624: 463-484. DOI: 10.1086/428890

Manousos, E., 2013. Mass and charge selfvariation: A common underlying cause for quantum phenomena and cosmological data. Progress Phys., 9: 73-141.

Peacock, J.A., S. Cole, P. Norberg, C.M. Baugh and J. Bland-Hawthorn et al., 2001. A measurement of the cosmological mass density from clustering in the $2 \mathrm{df}$ galaxy redshift survey. Nature, 410: 169-173. PMID: 11242069

Perlmutter, S., M.S. Turner and M. White, 1999. Constraining dark energy with type ia supernovae and Large-Scale structure. Phys. Rev. Lett., 83: 670673. DOI: 10.1103/PhysRevLett.83.670

Riess, A.G., A.V. Filippenko, P. Challis, A. Clocchiatti and A. Diercks et al., 1998. Observational evidence from supernovae for an accelerating universe and a cosmological constant. Astronomical J., 116: 10091038. DOI: $10.1086 / 300499$
Spergel, D.N., R. Bean, O. Dore, M.R. Nolta and C.L. Bennett et al., 2007. Three-Year Wilkinson Microwave Anisotropy Probe (WMAP) observations: Implications for cosmology. Astrophys. J. Suppl. Series, 170: $377-$ 408. DOI: $10.1086 / 513700$

Tzanavaris, P., J.K. Webb, M.T. Murphy, V.V. Flambaum and S.J. Curran, 2005. Limits on variations in fundamental constants from $21-\mathrm{cm}$ and ultraviolet Quasar absorption lines. Phys. Rev. Lett., 95: 41301-41304. PMID: 16090794

Walker, M.G., M.M. Olszewski, E.W. Penarrubia, J. Evans, N. Wyn et al., 2009. A universal mass profile for dwarf spheroidal galaxies? Astrophys. J., 704: 1274-1287.

Webb, J.K., J.A. King, M.T. Murphy, V.V. Flambaum and R.F. Carswell et al., 2011. Indications of a spatial variation of the fine structure constant. Phys. Rev. Lett., 107: 191101-191104. DOI: 10.1103/PhysRevLett.107.191101

Webb, J.K., V.V. Flambaum, C.W. Churchill, M.J. Drinkwater and J.D. Barrow, 1999. Search for time variation of the fine structure constant. Phys. Rev. Lett., 82: 884-887. DOI: 10.1103/PhysRevLett.82.884 